\title{
Aiding and abetting
}

DOI:

10.1038/nri1941

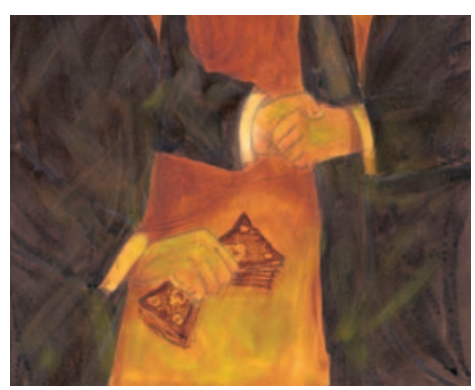

A new paper from the laboratory of Michael Lenardo reports that the movement of caspase- 8 to lipid rafts following T-cell receptor (TCR) stimulation is facilitated by tumournecrosis-factor-receptor-associated factor 6 (TRAF6).

TCR stimulation initiates a complex signalling pathway that is not yet fully understood but culminates in the activation of transcription factors, including nuclear factor- $\kappa \mathrm{B}$ $(\mathrm{NF}-\mathrm{\kappa B})$. Recent reports indicate that caspase-8, better known for its role in apoptosis, also has an important role in TCR-induced NF- $\kappa \mathrm{B}$ activation. In response to these reports, Lenardo and colleagues studied caspase- 8 and TRAF6 (a signal-transduction mediator that is known to activate transcription factors such as NF- $\mathrm{\kappa B}$ ) during TCR stimulation.

The authors found a direct interaction between caspase- 8 and TRAF6 following TCR stimulation. Two putative TRAF6-binding sites were identified in the sequence of caspase- 8 , and after mutating specific residues in these sites, the authors found that TRAF6 was unable to bind caspase- 8 , resulting in reduced TCR-induced NF- $\kappa B$ activation.

An essential step in TCR-mediated signal transduction is the enrichment of crucial molecules in plasma-membrane compartments known as lipid rafts. These molecules include caspase-8, TRAF6, protein kinase C- $\theta$ (PKC $\theta)$, caspase-recruitment domain membrane-associated guanylate kinase protein 1 (CARMA1) and B-cell lymphoma 10 (BCL-10). 'Knockdown' experiments indicated that caspase- 8 first forms a complex with BCL-10 in a PKCOdependent manner in the cytosol, leading to transient activation of caspase-8. Subsequently, TRAF6 and CARMA1 participate in the shuttling of activated caspase- 8 to lipid rafts, where (as part of a larger signalosome) caspase- 8 propagates NF- $\kappa B$ signalling.

These data indicate that the association of activated caspase- 8 with TRAF6 is crucial for the translocation of caspase- 8 to lipid rafts and for the subsequent TCR-induced activation of NF- $\kappa B$. Further questions remain, such as why transient activation of caspase- 8 is required for this process, but these results help to shed light on the complex molecular pathways that are involved in mounting a successful immune response.

Sharon Ahmad

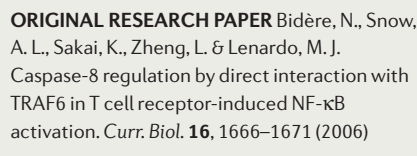

